Ann. Biol. anim. Bioch. Biophys., 1975, 15 (3), 6г5-6г7.

NOTE

\title{
ÉLIMINATION DE PÉNICILLINE LIBRE ET FIXÉE SOUS FORME DE CONJUGUÉS PENICILLOYL-PROTÉINES DANS LE LAIT DE VACHE APRÈS TRAITEMENT PAR VOIE INTRAMAMMAIRE
}

\author{
J. M. WAL et G. BORIES \\ Laboratoire de Recherches sur les Additifs alimentaires, I. N. R. A., \\ 180, chemin de Tournefeuille, \\ 31300 Toulouse
}

Des accidents allergiques, observés chez des consommateurs de lait provenant de vaches ayant reçu un traitement intramammaire à base de pénicilline, ont provoqué l'inquiétude des hygiénistes (GounelLe, I966, MALTEN, 1968). Les études du phénomène d'allergie à la pénicilline attribuent un rôle prépondérant aux conjugués pénicilloyl-protéines (JOSEPHSON, I960; Levine et OVARY, I96r). Les groupements pénicilloyl, issus des différentes pénicillines par rupture du cycle $\beta$-lactame, se fixent aux protéines par liaison covalente pour former des composés ayant perdu tout pouvoir antibactérien, mais à potentialité antigénique. Une autre voie de formation des conjugués pénicilloyl-protéines est décrite par l'intermédiaire de l'acide pénicillénique (Levine et OVARY, 1961 ; Doyle, I970). Si de tels composés sont susceptibles de se former chez l'animal à la suite de traitements thérapeutiques, ils passent inaperçus lors des contrôles d'hygiène des denrées d'origine animale, car ils ne peuvent être détectés par les méthodes de dosage microbiologique classique de la pénicilline. Nous nous sommes proposés de déterminer la nature, le taux et la persistance des résidus apparaissant dans le lait après administration par voie intraductale, d'une dose de pénicilline $\mathrm{G}$ correspondant à un traitement antimammite.

La pénicilline (benzyl pénicillinate de sodium cristallisé, Spécia) a été administrée par voie intraductale sous forme de pommade à $200000 \mathrm{UI} / \mathrm{g}$. Environ un million d'unités ont été injectées dans chaque trayon.

Le traitement a été effectué après la traite du matin. La recherche des résidus de pénicilline, libres ou conjugués, a été réalisée sur le lait de cette traite (témoin), et sur ceux des neuf traites suivantes.

La pénicilline libre, a été dosée par la méthode classique de diffusion sur gélose, utilisant Bacillus stéarothermophilus comme organisme test, suivant les instructions publiées sous l'égide du C. N. E. R. N. A. 
La détection et le dosage de la pénicilline fixée aux protéines du lait sous forme de conjugué pénicilloyl-protéine inactif du point de vue antibiotique, ont été réalisés au moyen d'une méthode radio-immunologique. Cette méthode, dont la mise en œuvre et les caractéristiques seront décrites dans une publication à paraître, est spécifique des groupements pénicilloyl et permet d'atteindre une limite de détection de l'ordre de quelques p.p.b. Elle est applicable directement aux laits, sans extraction préalable, après dilution convenable.

Les résultats sont rapportés dans le tableau ci-dessous :

Évolution des teneurs en pénicilline libre

et en groupements pénicilloyl de laits prélevés

à la suite d'un traitement intramammaire de pénicilline $G$

(quantités exprimées en microgrammes de pénicilline $\mathrm{G}$ ou d'acide benzyl pénicilloïque par gramme de lait)

\begin{tabular}{c|c|c}
$\begin{array}{c}\text { No de la traite } \\
\text { après traitement }\end{array}$ & $\begin{array}{c}\text { Teneur en } \\
\text { pénicilline G } \\
\text { (p.p.m.) }\end{array}$ & $\begin{array}{c}\text { Teneur en groupements } \\
\text { pénicilloyl } \\
\text { (p.p.m.) }\end{array}$ \\
& - & - \\
1 & $\vdots-\frac{1}{2}, 5$ & 1,5 \\
2 & 2,25 & 1,0 \\
3 & 0,6 & 0,13 \\
4 & 0,5 & 0,11 \\
5 & 0,55 & 0,022 \\
6 & 0,25 & 0,009 \\
7 & 0,13 & 0,004 \\
8 & 0,06 & 0,002 \\
9 & & \\
\hline
\end{tabular}

Des résidus de groupements pénicilloyl apparaissent donc à la suite d'un tel traitement. Leur teneur est très inférieure à celle des résidus de pénicilline libre et leur décroissance suit une courbe analogue. Ce n'est que 4 jours après le traitement que l'on cesse de détecter toute trace de pénicilloyl.

Afin d'éliminer tout risque d'interférence d'un excès de pénicilline libre sur le dosage des groupes pénicilloyl, les mesures ont été répétées après dialyse des laits pendant $48 \mathrm{~h}$ à $4^{\circ} \mathrm{C}$ contre de l'eau distillée. Les résultats obtenus après élimination de la pénicilline libre ne sont pas différents des précédents, ce qui montre qu'ils correspondent bien au dosage spécifique des conjugués pénicilloyl-protéines.

Reçu pour publication en avril 1975.

\section{REMERCIEMENTS}

Nous tenons à remercier M. Auclair et $\mathrm{M}^{\mathrm{me}}$ VAssal, Laboratoire de Bactériologie laitière, C. N. R. Z., $7835^{\circ}$ Jouy en Josas, de l'aide qu'ils nous ont apportée pour les dosages microbiologiques. 


\author{
SUMMARY \\ ELIMINATION OF FREE PENICILLIN AND PENCILLOYL-PROTEIN \\ CONJUGATES IN THE MILK OF COWS FOLLOWING INTRAMAMMARY \\ ADMINISTRATION OF PENICILLIN G
}

An intraductal injection of one million IU penicillin $G$ in each teat was administered to a cow. The milk was recovered during 9 consecutive milkıngs (4 days). Free penicillin residues were determined by a classical microbiological method. Penicilloyl-protein conjugates were detected and measured by a new specific radio-immunoassay. Free penicillin residues decrease rapidly from 22.5 p.p.m. to 0.66 p.p.m. on the $4^{\text {th }}$ day. Penicilloyl residues follow the same depleting process at a lower level; they appear already in the first milking following treatment (I.5 p.p.m.) and disappear completely only after 4 days. Evidence is given that penicilloyl residues exist as penicilloyl-protein conjugates.

\title{
RÉFÉRENCES BIBLIOGRAPHIQUES
}

C. N. E. R. N. A., I97I. Instructions sur la détection des antibiotiques dans les laits livrés par les producteurs. Rev. Lait. Française, 290, 587-593.

Doyle F. P., 1970. Proc. Symp. Advances in Penicillin Allergy, Rotterdam. Chemical back-ground, I5-23.

Gounelle H., Szakvary A., I966. Antibiotiques et Aliments. 1. Les accidents allergiques dus aux résidus. Bull. Acad. Med., 150, 76-82.

Josephson A.S., 1960. The development of antibodies to penicillin in rabbits. J. Exp. Med., 111, 6I I-6zO.

Levine B. B., Ovary Z., I96I. Studies on the mechanism of the formation of the Penicillin Antigen (III). J. Exp. Med., 114, 875-904.

Malten K. E., i966. Proc. 5th Symp. Group. Europ. Nutr. Jouy en Josas. Antibiotics in Agriculture. Bibl. Nutritio et Dieta, 10, $184^{-194 . ~}$ 